\title{
Coupled fixed points for multivalued mappings in fuzzy metric spaces
}

\author{
Zheyong Qiu and Shihuang Hong*
}

"Correspondence:

hongshh@hotmail.com

Institute of Applied Mathematics

and Engineering Computations,

Hangzhou Dianzi University,

Hangzhou, 310018, People's

Republic of China

\begin{abstract}
In this paper, we establish two coupled fixed point theorems for multivalued nonlinear contraction mappings in partially ordered fuzzy metric spaces. The theorems presented extend some corresponding results due to ordinary metric spaces. An example is given to illustrate the usability of our results.
\end{abstract}

Keywords: coupled fixed point; multivalued contractive mapping; fuzzy metric space; partially ordered set

\section{Introduction}

In 1969, Nadler [1] extended the famous Banach contraction principle from single-valued mappings to multivalued mappings and proved the existence of fixed points for contractive multivalued mappings in complete metric spaces. Since then, the existence of fixed points for various multivalued contractive mappings has been studied by many authors under different conditions. For details, we refer to [2-10] and the references therein. For instance, in [3] Ćirić has proved a fixed point theorem for the single-valued mappings satisfying some contractive condition. Samet and Vetro [4] extended this result to multivalued mappings and proved the existence of a coupled fixed point theorem for the multivalued contraction.

One of the most important problems in fuzzy topology is to obtain an appropriate concept of fuzzy metric spaces. This problem has been investigated by many authors from different points of view. In particular, George and Veeramani [11,12] introduced and studied the notion of fuzzy metric $M$ on a set $X$ with the help of continuous $t$-norms introduced in [13], and from now on, when we talk about fuzzy metrics, we refer to this type. Fuzzy metric spaces have many applications. In particular, on the fuzzy metric space, by using some topological properties induced by this kind of fuzzy metrics, there are several fixed point results established. Some instances of these works are in [14-23]. In fact, fuzzy fixed point results are more versatile than the regular metric fixed point results. This is due to the flexibility which the fuzzy concept inherently possesses. For example, the Banach contraction mapping principle has been extended in fuzzy metric spaces in two inequivalent ways in $[17,24]$. Fuzzy fixed point theory has a developed literature and can be regarded as a subject in its own right (see [16]).

In recent times, the existence of common or coupled fixed points of a fuzzy version for multiple mappings has attracted much attention. We mention that the coupled fixed point results were proved by Sedghi et al. [15], which is a fuzzy version of the result of [25]. Choudhury [16] further extended the result of [25] and provided the existence results of coupled coincidence points for compatible mappings in partially ordered fuzzy 
metric spaces. After that common coupled fixed point results in fuzzy metric spaces were established by $\mathrm{Hu}$ [19]. However, to the best of our knowledge, few papers were devoted to fixed point problems of multivalued mappings in fuzzy metric spaces (see [26, 27]).

The aim of this paper is to present two new coupled fixed point theorems for two multivalued mappings in the fuzzy metric space. The idea of the present paper originates from the study of an analogous problem examined by Samet et al. [4] in regular partially ordered metric spaces due to Ćirić [3]. Our results give a significant extension of some corresponding results. An example is also given to illustrate the suitability of our results.

\section{Preliminaries}

To set up our main results in the sequel, we recall some necessary definitions and preliminary concepts in this section.

Definition 2.1 [13] The binary operation $*:[0,1] \times[0,1] \rightarrow[0,1]$ is called a continuous $t$-norm if the following properties are satisfied:

(T1) $a * 1=a$ for all $a \in[0,1]$,

(T2) $a * b \leq c * d$ whenever $a \leq c$ and $b \leq d$ for each $a, b, c, d \in[0,1]$,

(T3) $*$ is continuous, associative and commutative.

In this sequel, we further assume that $*$ satisfies

(T4) $a * b \geq a b$ for all $a, b \in[0,1]$.

For examples of $t$-norm satisfying the conditions (T1)-(T4), we enumerate $a * b=a b$, $a * b=\min \{a, b\}$ and $a * b=a b / \max \{a, b, \lambda\}$ for $0<\lambda<1$, respectively.

Definition 2.2 [11] The triplet $(X, M, *)$ is said to be a fuzzy metric space if $X$ is an arbitrary nonempty set, $*$ is a continuous $t$-norm and $M$ is a fuzzy set on $X \times X \times(0,+\infty)$ satisfying the following conditions for all $x, y, z \in X, s, t>0$ :

(F1) $M(x, y, t)>0$,

(F2) $M(x, y, t)=1$ for all $t>0$ if and only if $x=y$,

(F3) $M(x, y, t)=M(y, x, t)$,

(F4) $M(x, y, t) * M(y, z, s) \leq M(x, z, t+s)$ and

(F5) $M(x, y, \cdot):(0,+\infty) \rightarrow[0,1]$ is continuous.

In this sense, $(M, *)$ is called a fuzzy metric on $X$.

Example 2.3 [11] Let $X$ be the set of all real numbers and $d$ be the Euclidean metric. Let $a * b=\min \{a, b\}$ for all $a, b \in[0,1]$. For each $t>0, x, y \in X$, let $M(x, y, t)=\frac{t}{t+d(x, y)}$. Then $(X, M, *)$ is a fuzzy metric space.

Let $(X, M, *)$ be a fuzzy metric space. For $t>0$ and $0<r<1$, the open ball $B(x, t, r)$ with center $x \in X$ is defined by

$$
B(x, t, r)=\{y \in X: M(x, y, t)>1-r\} .
$$

A subset $A \subset X$ is called open if for each $x \in A$, there exist $t>0$ and $0<r<1$ such that $B(x, t, r) \subset A$. Let $\mathcal{T}$ denote the family of all open subsets of $X$. Then $\mathcal{T}$ is a topology on $X$ induced by the fuzzy metric $(M, *)$. This topology is metrizable (see [12]). Therefore, a closed subset $B$ of $X$ is equivalent to $x \in B$ if and only if there exists a sequence $\left\{x_{n}\right\} \subset B$ 
such that $\left\{x_{n}\right\}$ topologically converges to $x$. In fact, the topological convergence of sequences can be indicated by the fuzzy metric as follows.

Definition 2.4 [11] Let $(X, M, *)$ be a fuzzy metric space.

(i) A sequence $\left\{x_{n}\right\}$ in $X$ is said to be convergent to a point $x \in X$ if $\lim _{n \rightarrow \infty} M\left(x_{n}, x, t\right)=1$ for any $t>0$.

(ii) A sequence $\left\{x_{n}\right\}$ in $X$ is called a Cauchy sequence if $\lim _{n \rightarrow \infty} M\left(x_{n+p}, x_{n}, t\right)=1$ for any $t>0$ and a positive integer $p$.

(iii) A fuzzy metric space $(X, M, *)$, in which every Cauchy sequence is convergent, is said to be complete.

By $C B(X)$ we denote the collection consisting of all nonempty closed subsets of $X$. For $C \in C B(X)$ and $x \in X$, we define

$$
M^{\nabla}(C, x, t)=M^{\nabla}(x, C, t)=\sup \{M(z, x, t): z \in C\} .
$$

Lemma 2.5 If $A \in C B(X), x \in A$ if and only if $M^{\nabla}(A, x, t)=1$ for all $t>0$.

Proof Since $M^{\nabla}(A, x, t)=\sup \{M(x, y, t): y \in A\}=1$, there exists a sequence $\left\{y_{n}\right\} \subset A$ such that $M\left(x, y_{n}, t\right)>1-\frac{1}{n}$. Let $n \rightarrow \infty$, we have $y_{n} \rightarrow x$. From $A \in C B(X)$ it follows that $x \in A$. Conversely, if $x \in A$, we have $M^{\nabla}(A, x, t)=\sup \{M(x, y, t): y \in A\} \geq M(x, x, t)=1$ for any $t>0$. This implies that $M^{\nabla}(A, x, t)=1$ for all $t>0$.

Definition 2.6 An element $(x, y) \in X \times X$ is a coupled fixed point of $F: X \times X \rightarrow C B(X)$ if

$$
x \in F(x, y) \text { and } y \in F(y, x) .
$$

At the end of this section, we introduce the following necessary notions.

The function $f: X \times X \times(0, \infty) \rightarrow(0, \infty)$ is said to be uniformly upper semi-continuous with respect to $t \in(0, \infty)$ on $X \times X$ if $\lim _{n \rightarrow \infty}\left(x_{n}, y_{n}\right)=(x, y)$ implies that $f(x, y, t) \geq$ $\limsup _{n \rightarrow \infty} f\left(x_{n}, y_{n}, t\right)$ for $\left\{x_{n}\right\},\left\{y_{n}\right\} \subset X$ and all $t \in(0, \infty)$.

Let $X$ be a nonempty set endowed with a partial order $\preceq$ and let $G: X \rightarrow X$ be a given mapping. We recall the set

$$
\Delta=\{(x, y) \in X \times X: G x \preceq G y\}
$$

and the binary relation $\mathcal{R}$ on $C B(X)$ defined by

$$
A \mathcal{R} B \quad \Leftrightarrow \quad A \times B \subset \Delta \quad \text { for } A, B \in C B(X) .
$$

Definition 2.7 Let $F: X \times X \rightarrow C B(X)$ be a given mapping. We say that $F$ is a $\Delta$ symmetric mapping if and only if

$$
(x, y) \in \Delta \quad \Rightarrow \quad F(x, y) \mathcal{R} F(y, x) .
$$




\section{Main results}

In order to prove our main results, we need the following hypothesis.

(H) The function $f: X \times X \times(0, \infty) \rightarrow(0,2]$ defined by

$$
f(x, y, t)=M^{\nabla}(x, F(x, y), t)+M^{\nabla}(y, F(y, x), t)
$$

with $F: X \times X \rightarrow C B(X)$ is uniformly upper semi-continuous with respect to $t \in(0, \infty)$ on $X \times X$.

Theorem 3.1 Let $(X, M, *)$ be a complete fuzzy metric space with the partial order $\preceq$ and $\left(x_{0}, y_{0}\right) \in \Delta$. Let $F: X \times X \rightarrow C B(X)$ be a $\Delta$-symmetric mapping and satisfy that

(i) the condition $(\mathrm{H})$ holds and

(ii) for any $(x, y) \in \Delta$, if $g(x, y)=\inf _{t>0} f(x, y, t)<2$, then there exist $u \in F(x, y)$ and $v \in F(y, x)$ with

$$
\sqrt{\varphi(g(x, y))}[M(x, u, t)+M(y, v, t)] \geq f(x, y, t)
$$

such that

$$
f(u, v, t) \geq \varphi(g(x, y))[M(x, u, t)+M(y, v, t)]
$$

where, $t>0$, the function $\varphi:[0,2] \rightarrow[1,+\infty)$ is nonincreasing, $\varphi(r)>1$ for $0 \leq r<2$ and $\lim _{r \rightarrow 2^{-}} \varphi(r)>1$.

Then $F$ admits a coupled fixed point on $X \times X$.

Proof If $g(x, y)=2$, then it is easy to see that $(x, y) \in X \times X$ is a coupled fixed point of $F$. Otherwise, $g(x, y)<2$ and the definition of $\varphi$ guarantees that $\varphi(g(x, y))>1$ for each $(x, y) \in$ $X \times X$. By virtue of the definition of $M^{\nabla}$, there exist $u \in F(x, y)$ and $v \in F(y, x)$ for any given $(x, y) \in X \times X$ such that

$$
\sqrt{\varphi(g(x, y))} M(x, u, t) \geq M^{\nabla}(x, F(x, y), t), \quad \sqrt{\varphi(g(x, y))} M(y, v, t) \geq M^{\nabla}(y, F(y, x), t) .
$$

This implies that, for each $(x, y) \in X \times X$, there exist $u \in F(x, y)$ and $v \in F(y, x)$ such that (2) holds. Thus, for arbitrarily given $\left(x_{0}, y_{0}\right) \in \Delta$, either $g\left(x_{0}, y_{0}\right)=2$, then we get our desired result, or $g\left(x_{0}, y_{0}\right)<2$. In this case, by the condition (ii), we can choose $x_{1} \in F\left(x_{0}, y_{0}\right)$ and $y_{1} \in F\left(y_{0}, x_{0}\right)$ such that

$$
\begin{aligned}
& \sqrt{\varphi\left(g\left(x_{0}, y_{0}\right)\right)}\left[M\left(x_{0}, x_{1}, t\right)+M\left(y_{0}, y_{1}, t\right)\right] \geq f\left(x_{0}, y_{0}, t\right), \\
& \varphi\left(g\left(x_{0}, y_{0}\right)\right)\left[M\left(x_{0}, x_{1}, t\right)+M\left(y_{0}, y_{1}, t\right)\right] \leq f\left(x_{1}, y_{1}, t\right) .
\end{aligned}
$$

From (4) and (5) it yields that

$$
\begin{aligned}
f\left(x_{1}, y_{1}, t\right) & \geq \sqrt{\varphi\left(g\left(x_{0}, y_{0}\right)\right)}\left\{\sqrt{\varphi\left(g\left(x_{0}, y_{0}\right)\right)}\left[M\left(x_{0}, x_{1}, t\right)+M\left(y_{0}, y_{1}, t\right)\right]\right\} \\
& \geq \sqrt{\varphi\left(g\left(x_{0}, y_{0}\right)\right)} f\left(x_{0}, y_{0}, t\right) .
\end{aligned}
$$


Note that $F$ is a $\Delta$-symmetric mapping. By Definition 2.7 we have $F\left(x_{0}, y_{0}\right) \mathcal{R} F\left(y_{0}, x_{0}\right)$, which further implies $\left(x_{1}, y_{1}\right) \in \Delta$. If $g\left(x_{1}, y_{1}\right)=2$, then $\left(x_{1}, y_{1}\right)$ is a coupled fixed point of $F$ and our desired result comes out. Otherwise, $g\left(x_{1}, y_{1}\right)<2$, which implies that $\varphi\left(g\left(x_{1}, y_{1}\right)\right)>1$. Again, the condition (ii) guarantees that there exist $x_{2} \in F\left(x_{1}, y_{1}\right)$ and $y_{2} \in F\left(y_{1}, x_{1}\right)$ such that

$$
\begin{aligned}
& \sqrt{\varphi\left(g\left(x_{1}, y_{1}\right)\right)}\left[M\left(x_{1}, x_{2}, t\right)+M\left(y_{1}, y_{2}, t\right)\right] \geq f\left(x_{1}, y_{1}, t\right), \\
& \varphi\left(g\left(x_{1}, y_{1}\right)\right)\left[M\left(x_{1}, x_{2}, t\right)+M\left(y_{1}, y_{2}, t\right)\right] \leq f\left(x_{2}, y_{2}, t\right) .
\end{aligned}
$$

Hence, we obtain $\left(x_{2}, y_{2}\right) \in \Delta$ and

$$
f\left(x_{2}, y_{2}, t\right) \geq \sqrt{\varphi\left(g\left(x_{1}, y_{1}\right)\right)} f\left(x_{1}, y_{1}, t\right)
$$

Continuing this process, we can choose the sequences $\left\{x_{n}\right\},\left\{y_{n}\right\} \subset X$ such that either $g\left(x_{n}, y_{n}\right)=2$, which implies that $\left(x_{n}, y_{n}\right)$ is a coupled fixed point of $F$, or $g\left(x_{n}, y_{n}\right)<2$, which implies $\varphi\left(g\left(x_{n}, y_{n}\right)\right)>1$. In this case, we have

$$
\begin{aligned}
& \left(x_{n}, y_{n}\right) \in \Delta, \quad x_{n+1} \in F\left(x_{n}, y_{n}\right), \quad y_{n+1} \in F\left(y_{n}, x_{n}\right), \\
& \sqrt{\varphi\left(g\left(x_{n}, y_{n}\right)\right)}\left[M\left(x_{n}, x_{n+1}, t\right)+M\left(y_{n}, y_{n+1}, t\right)\right] \geq f\left(x_{n}, y_{n}, t\right)
\end{aligned}
$$

for $n=0,1,2, \ldots$ and

$$
f\left(x_{n+1}, y_{n+1}, t\right) \geq \sqrt{\varphi\left(g\left(x_{n}, y_{n}\right)\right)} f\left(x_{n}, y_{n}, t\right) \quad \text { for } n=0,1,2, \ldots
$$

It follows that the sequence $\left\{f\left(x_{n}, y_{n}, t\right)\right\}$ is increasing from (8) and upper bounded from the definition of $f$. Therefore, this deduces $\left\{f\left(x_{n}, y_{n}, t\right)\right\}$ is convergent, say,

$$
\lim _{n \rightarrow \infty} f\left(x_{n}, y_{n}, t\right)=\alpha(t)
$$

We assert $\alpha(t) \equiv 2$ for $t>0$. Suppose that this is not true, then there exists $t_{0}>0$ such that $0<\alpha\left(t_{0}\right)<2$. Note that $g\left(x_{n}, y_{n}\right) \leq f\left(x_{n}, y_{n}, t_{0}\right)$, we have $\varphi\left(g\left(x_{n}, y_{n}\right)\right) \geq \varphi\left(f\left(x_{n}, y_{n}, t_{0}\right)\right)$ for each $n \in \mathbb{N}$ since $\varphi$ is nonincreasing. By means of this, taking the limit on both sides of (8) with $t=t_{0}$ and having in mind the assumptions of $\varphi$, we have

$$
\begin{aligned}
\alpha\left(t_{0}\right) & \geq \lim _{f\left(x_{n}, y_{n}, t_{0}\right) \rightarrow \alpha\left(t_{0}\right)} \sqrt{\varphi\left(g\left(x_{n}, y_{n}\right)\right)} f\left(x_{n}, y_{n}, t_{0}\right) \\
& \geq \lim _{f\left(x_{n}, y_{n}, t_{0}\right) \rightarrow \alpha\left(t_{0}\right)^{-}} \sqrt{\varphi\left(f\left(x_{n}, y_{n}, t_{0}\right)\right)} \alpha\left(t_{0}\right)>\alpha\left(t_{0}\right)
\end{aligned}
$$

a contradiction. Thus $\alpha(t) \equiv 2$ for all $t>0$.

We are in a position to verify that both $\left\{x_{n}\right\}$ and $\left\{y_{n}\right\}$ are Cauchy sequences in $(X, M, *)$. Let

$$
\beta=\lim _{f\left(x_{n}, y_{n}, t\right) \rightarrow 2^{-}} \sqrt{\varphi\left(g\left(x_{n}, y_{n}\right)\right)} .
$$


Note that $\left\{g\left(x_{n}, y_{n}\right)\right\}$ is nondecreasing, we obtain that $\left\{\varphi\left(g\left(x_{n}, y_{n}\right)\right)\right\}$ is nonincreasing. Hence, $\beta$ exists. Since $\varphi\left(g\left(x_{n}, y_{n}\right)\right)>1$, the properties of $\varphi$ guarantee that $\beta>1$. Let the real number $p$ with $\beta>p>1$ and $p^{2}>\beta$ be any fixed. For any $\varepsilon>0$ with $\varepsilon<\min \left\{1, p^{2}-\beta\right\}$, there exists $n_{2} \in \mathbb{N}$ such that

$$
\beta+\varepsilon \geq \sqrt{\varphi\left(g\left(x_{n}, y_{n}\right)\right)} \text { for each } n \geq n_{2} \text {. }
$$

On the other hand, we easily see that there exists $n_{1} \in \mathbb{N}$ such that

$$
\sqrt{\varphi\left(g\left(x_{n}, y_{n}\right)\right)}>p \quad \text { for each } n \geq n_{1} \text {. }
$$

Substituting this inequality in (8), we have

$$
f\left(x_{n+1}, y_{n+1}, t\right)>p f\left(x_{n}, y_{n}, t\right) \text { for all } t>0 \text { and } n \geq n_{1} \text {. }
$$

For any $m \in \mathbb{N}$, we inductively obtain

$$
f\left(x_{n+m}, y_{n+m}, t\right)>p^{m} f\left(x_{n}, y_{n}, t\right) \quad \text { for all } t>0 \text { and } n \geq n_{1} \text {. }
$$

Let $n_{0} \geq \max \left\{n_{1}, n_{2}\right\}$. By means of this, together with (7) and (9) with $n=n_{0}$, one has

$$
(\beta+\varepsilon)\left[M\left(x_{n_{0}+m}, x_{n_{0}+m+1}, t\right)+M\left(y_{n_{0}+m}, y_{n_{0}+m+1}, t\right)\right]>p^{m} f\left(x_{n_{0}}, y_{n_{0}}, t\right)
$$

By virtue of $\alpha(t) \equiv 2$, we have $f\left(x_{n_{0}}, y_{n_{0}}, t\right) \geq 2-\varepsilon$ when $n_{0}$ is large enough. In addition, the hypothesis of $\varepsilon$ implies that $p^{m} \geq \beta+\varepsilon$ for $m \geq 2$. Consequently, we can choose that $n_{0} \in \mathbb{N}$ is large enough such that

$$
M\left(x_{n}, x_{n+1}, t\right)+M\left(y_{n}, y_{n+1}, t\right)>2-\varepsilon \text { for any } t>0 \text { and } n \geq n_{0},
$$

which implies that, by the arbitrariness of $\varepsilon$,

$$
M\left(x_{n}, x_{n+1}, t\right)>1-\varepsilon, \quad M\left(y_{n}, y_{n+1}, t\right)>1-\varepsilon \quad \text { for any } t>0 \text { and } n \geq n_{0} .
$$

In order to prove that $\left\{x_{n}\right\}$ and $\left\{y_{n}\right\}$ are Cauchy sequences, for any given $\epsilon>0$, it is sufficient to check

$$
M\left(x_{n}, x_{m}, t\right)>1-\epsilon, \quad M\left(y_{n}, y_{m}, t\right)>1-\epsilon
$$

for any $m>n \geq n_{0}+2$ and $t>0$. Virtually, for any $n \geq n_{0}+2$, if $m=n+1$, then (11) is valid by (10) with $\varepsilon \leq \epsilon$. Inductively suppose that (11) is valid for $m=k>n$, each $t>0$ and $n \geq n_{0}+2$. Then, in view of the hypotheses (F4) and (T4), we have

$$
M\left(x_{n}, x_{k+1}, t\right) \geq M\left(x_{n}, x_{k}, t / 2\right) * M\left(x_{k}, x_{k+1}, t / 2\right) \geq M\left(x_{n}, x_{k}, t / 2\right) M\left(x_{k}, x_{k+1}, t / 2\right) .
$$

Now, the inductive assumption shows that $M\left(x_{n}, x_{k}, t / 2\right)>1-\epsilon$ and the first inequality in (10) guarantees $M\left(x_{k}, x_{k+1}, t / 2\right)>1-\varepsilon$. Hence we have

$$
M\left(x_{n}, x_{k+1}, t\right)>(1-\epsilon)(1-\varepsilon) .
$$


Let $\varepsilon \rightarrow 0$, we have $M\left(x_{n}, x_{k+1}, t\right)>1-\epsilon$. By induction, we get that the first inequality in (11) is true. The proof of the second inequality in (11) is analogous.

Finally, by means of the completeness of $(X, M, *)$, there exists $z=\left(z_{1}, z_{2}\right) \in X \times X$ such that

$$
\lim _{n \rightarrow \infty} x_{n}=z_{1} \text { and } \quad \lim _{n \rightarrow \infty} y_{n}=z_{2}
$$

In what follows, we prove that $z$ is a coupled fixed point of $F$. Since $f$ is upper semicontinuous, from $\lim _{n \rightarrow \infty} f\left(x_{n}, y_{n}, t\right)=\alpha(t) \equiv 2$ we get

$$
2 \geq f(z)=M^{\nabla}\left(z_{1}, F\left(z_{1}, z_{2}\right), t\right)+M^{\nabla}\left(z_{2}, F\left(z_{2}, z_{1}\right), t\right) \geq \limsup _{n \rightarrow \infty} f\left(x_{n}, y_{n}, t\right)=2 .
$$

This implies $M^{\nabla}\left(z_{1}, F\left(z_{1}, z_{2}\right), t\right)+M^{\nabla}\left(z_{2}, F\left(z_{2}, z_{1}\right), t\right)=2$; moreover, we have

$$
M^{\nabla}\left(z_{1}, F\left(z_{1}, z_{2}\right), t\right)=1 \quad \text { and } \quad M^{\nabla}\left(z_{2}, F\left(z_{2}, z_{1}\right), t\right)=1 .
$$

From Lemma 2.5 it follows that $z_{1} \in F\left(z_{1}, z_{2}\right)$ and $z_{2} \in F\left(z_{2}, z_{1}\right)$, that is, $z=\left(z_{1}, z_{2}\right)$ is a coupled fixed point of $F$. The proof is completed.

Theorem 3.2 Let $(X, M, *)$ be a complete fuzzy metric space endowed with a partial order $\preceq, \Delta \neq \emptyset$, and let $F: X \times X \rightarrow C B(X)$ be a $\Delta$-symmetric multivalued mapping satisfying that

(i) the $(\mathrm{H})$ holds and

(ii) for any $(x, y) \in \Delta$, there exist $u \in F(x, y)$ and $v \in F(y, x)$ with $h(x, y, u, v)<2$ and

$$
\sqrt{\varphi(h(x, y, u, v))}[M(x, u, t)+M(y, v, t)] \geq f(x, y, t)
$$

such that

$$
f(u, v, t) \geq \varphi(h(x, y, u, v))[M(x, u, t)+M(y, v, t)]
$$

where $h(x, y, u, v)=\inf _{t>0}\{M(x, u, t)+M(t, v, t)\}<2$ for $x, y, u, v \in X$, the function $\varphi:[0,2] \rightarrow[1,+\infty)$ is nonincreasing and satisfies that $\varphi(r)>1$ for $0 \leq r<2$ and $\liminf _{r \rightarrow s^{-}} \varphi(r)>1$ for each $s \in(0,2)$.

Then $F$ admits a coupled fixed point on $X \times X$.

Proof If $h(x, y, u, v)=2$, then it is easy to see that $x=u$ and $y=v$. In this case, $(x, y)$ is a coupled fixed point of $F$ if $u \in F(x, y)$ and $v \in F(y, x)$. Otherwise, $h(x, y, u, v)<2$ and the definition of $\varphi$ guarantees that $\varphi(h(x, y, u, v))>1$ for each $(x, y) \in X \times X$. As an analogy of the argument of Theorem 3.1, we can construct the sequences $\left\{x_{n}\right\},\left\{y_{n}\right\} \subset X$ such that either $h\left(x_{n}, y_{n}, x_{n+1}, y_{n+1}\right)=2$, which implies that $\left(x_{n}, y_{n}\right)$ is a coupled fixed point of $F$, or $h\left(x_{n}, y_{n}, x_{n+1}, y_{n+1}\right)<2$, which implies $\varphi\left(h\left(x_{n}, y_{n}, x_{n+1}, y_{n+1}\right)\right)>1$. In this case, we have

$$
\begin{aligned}
& \left(x_{n}, y_{n}\right) \in \Delta, \quad x_{n+1} \in F\left(x_{n}, y_{n}\right), \quad y_{n+1} \in F\left(y_{n}, x_{n}\right), \\
& \sqrt{\varphi\left(h\left(x_{n}, y_{n}, x_{n+1}, y_{n+1}\right)\right)}\left[M\left(x_{n}, x_{n+1}, t\right)+M\left(y_{n}, y_{n+1}, t\right)\right] \geq f\left(x_{n}, y_{n}, t\right)
\end{aligned}
$$


for $n=0,1,2, \ldots$ and

$$
f\left(x_{n+1}, y_{n+1}, t\right) \geq \sqrt{\varphi\left(h\left(x_{n}, y_{n}, x_{n+1}, y_{n+1}\right)\right)} f\left(x_{n}, y_{n}, t\right) \quad \text { for } n=0,1,2, \ldots
$$

Again, (16) guarantees that the sequence $\left\{f\left(x_{n}, y_{n}, t\right)\right\}$ is increasing and upper bounded. Therefore, there exists $\alpha:(0, \infty) \rightarrow(0,2]$ such that

$$
\lim _{n \rightarrow \infty} f\left(x_{n}, y_{n}, t\right)=\alpha(t) \quad \text { for } t>0
$$

On the other hand, note that the sequence $\left\{h_{n}\right\}$ with $h_{n}=\left\{h\left(x_{n}, y_{n}, x_{n+1}, y_{n+1}\right)\right\}$ is bounded, we have

$$
\limsup _{n \rightarrow \infty} h_{n}=\eta
$$

for some $\eta \in(0,2]$. By virtue of (14), combining (1), we have

$$
M\left(x_{n}, x_{n+1}, t\right)+M\left(y_{n}, y_{n+1}, t\right) \leq f\left(x_{n}, y_{n}, t\right)
$$

for any $n \in \mathbb{N}$ and $t>0$, which implies that $h_{n} \leq f\left(x_{n}, y_{n}, t\right)$. Moreover, one has $\eta \leq \alpha(t)$ for all $t>0$.

In what follows, we prove that $\eta=2$. To this end, we first prove $\alpha(t) \equiv 2$ for all $t>0$. Suppose, to the contrary, that there exists $t_{0}>0$ such that $\alpha\left(t_{0}\right)<2$. The monotonicity of the sequence $\left\{f\left(x_{n}, y_{n}, t_{0}\right)\right\}$ and $h_{n} \leq f\left(x_{n}, y_{n}, t_{0}\right)$ yields that $\eta \leq \alpha\left(t_{0}\right)$ and $\varphi\left(h_{n}\right)>1$ for all $n \in \mathbb{N}$. In addition, there exists the subsequence of $\left\{h_{n}\right\},\left\{h_{n_{k}}\right\}$, such that $\lim _{k \rightarrow \infty} h_{n_{k}}=\eta^{-}<2$. In view of the hypothesis of $\varphi$, we have $\liminf _{k \rightarrow \infty} \sqrt{\varphi\left(h_{n_{k}}\right)}>1$. By means of this and the property of $\varphi$, taking the limit on both sides of the following inequality:

$$
f\left(x_{n_{k}+1}, y_{n_{k}+1}, t_{0}\right) \geq \sqrt{\varphi\left(h_{n_{k}}\right)} f\left(x_{n_{k}}, y_{n_{k}}, t_{0}\right),
$$

we obtain $\alpha\left(t_{0}\right)=\liminf _{k \rightarrow \infty} f\left(x_{n_{k}+1}, y_{n_{k}+1}, t_{0}\right) \geq \liminf _{k \rightarrow \infty}\left[\sqrt{\varphi\left(h_{n_{k}}\right)} f\left(x_{n_{k}}, y_{n_{k}}, t_{0}\right)\right]>\alpha\left(t_{0}\right)$, a contradiction. Hence, $\alpha(t) \equiv 2$. If $\eta<2$, repeating the above proceeding, we can obtain the contradiction of $2>2$. Consequently, $\eta=2$.

Finally, by $\eta=2$, it is easy to see that the sequences $\left\{x_{n_{k}}\right\}$ and $\left\{y_{n_{k}}\right\}$ satisfy (10). Following the lines of the arguments of Theorem 3.1, we can obtain that $\left\{x_{n_{k}}\right\}$ and $\left\{y_{n_{k}}\right\}$ are Cauchy sequences. The rest of the proof is the same as that of Theorem 3.1. This completes our proof.

\section{An example}

In this section, we conclude the paper with the following example.

Example 4.1 Let $X=[1, \infty), a * b=a b$ for all $a, b \in[0,1]$ and

$$
M(x, y, t)= \begin{cases}1, & x=y, \\ \frac{1}{x y}, & x \neq y .\end{cases}
$$


Then $(X, M, *)$ is a fuzzy metric space (see [28]). Let $X$ be endowed with the usual order $\leq$. Define the function $G: X \rightarrow X$ by $G(x)=c$ for any $x \in X$, where $c$ is a positive constant. Then $\Delta=(1, \infty) \times(1, \infty)$. Define the multivalued function $F: X \times X \rightarrow C B(X)$ by

$$
F(x, y)=\left\{\begin{array}{ll}
{\left[\frac{\sqrt{y}+2}{3}, \frac{\sqrt{x+y}+2}{3}\right],} & 1 \leq y \leq 3, \\
\left\{\frac{\sqrt{y}+2}{3}\right\}, & y>3,
\end{array} \quad \text { for all } x \in X\right.
$$

Conclusion $F$ admits a coupled fixed point in $X \times X$.

Proof It is not hard to see that $X$ is complete and $F$ is a $\Delta$-symmetric mapping. Moreover,

$$
M^{\nabla}(x, F(x, y), t)=\frac{3}{x(\sqrt{y}+2)}, \quad M^{\nabla}(y, F(y, x), t)=\frac{3}{(\sqrt{x}+2) y}
$$

Adding the above two formulae together, we obtain

$$
f(x, y, t)=\frac{3}{x(\sqrt{y}+2)}+\frac{3}{(\sqrt{x}+2) y} \quad \text { for }(x, y, t) \in X \times X \times(0,+\infty) .
$$

$f$ is obviously uniformly upper semi-continuous with respect to $t \in(0,+\infty)$, i.e., $(\mathrm{H})$ is satisfied. Let the function $\varphi:[0,2] \rightarrow[1, \infty)$ be defined by

$$
\varphi(t)=2-\frac{t}{2}, \quad t \in(0,2]
$$

For any $x, y \in X$, we take $u=\frac{\sqrt{y}+2}{3}, v=\frac{\sqrt{x}+2}{3}$, then $u \in F(x, y), v \in F(y, x)$ and

$$
M(x, u, t)=\frac{3}{x(\sqrt{y}+2)}, \quad M(y, v, t)=\frac{3}{(\sqrt{x}+2) y} .
$$

Therefore, $u, v$ satisfy the inequality (2) and

$$
\begin{aligned}
f(u, v, t) & =\frac{3}{u(\sqrt{v}+2)}+\frac{3}{(\sqrt{u}+2) v} \\
& =\frac{9}{(\sqrt{y}+2)} \frac{1}{\sqrt{\frac{\sqrt{x}+2}{3}+2}}+\frac{9}{(\sqrt{x}+2)} \frac{1}{\sqrt{\frac{\sqrt{y}+2}{3}+2}} \\
& =\frac{3}{x(\sqrt{y}+2)} \cdot \frac{3 \sqrt{3} x}{\sqrt{\sqrt{x}+2+2 \sqrt{3}}}+\frac{3}{y(\sqrt{x}+2)} \cdot \frac{3 \sqrt{3} y}{\sqrt{\sqrt{y}+2+2 \sqrt{3}}} \\
& \geq 2\left[\frac{3}{x(\sqrt{y}+2)}+\frac{3}{(\sqrt{x}+2) y}\right] \\
& \geq \varphi(g(x, y))[M(x, u, t)+M(y, v, t)] .
\end{aligned}
$$

This yields that the inequality (3) is valid. (3) is obviously true if $x=y$. Now Theorem 3.1 guarantees that $F$ admits a coupled fixed point on $X \times X$. 


\section{Competing interests}

The authors declare that they have no competing interests.

\section{Authors' contributions}

Both authors read and approved the final manuscript.

\section{Acknowledgements}

The authors wish to express their hearty thanks to the anonymous referees for their valuable suggestions and comments. Supported by Natural Science Foundation of Zhejiang Province (LY12A01002).

\section{Received: 11 January 2013 Accepted: 4 June 2013 Published: 25 June 2013}

\section{References}

1. Nadler, SB Jr.: Multi-valued contraction mappings. Pac. J. Math. 30, 475-488 (1969)

2. Ćirić, LB: Fixed point theorems for multi-valued contractions in complete metric spaces. J. Math. Anal. Appl. 348, 499-507 (2008)

3. Ćirić, LB: Multi-valued nonlinear contraction mappings. Nonlinear Anal. 71, 2716-2723 (2009)

4. Samet, B, Vetro, C: Coupled fixed point theorems for multi-valued nonlinear contraction mappings in partially ordered metric spaces. Nonlinear Anal. 74, 4260-4268 (2011)

5. Hong, SH: Fixed points for mixed monotone multivalued operators in Banach spaces with applications. J. Math. Anal. Appl. 337, 333-342 (2008)

6. Hong, SH, Guan, D, Wang, L: Hybrid fixed points of multivalued operators in metric spaces with applications. Nonlinear Anal. 70, 4106-4117 (2009)

7. Hong, SH: Fixed points of multivalued operators in ordered metric spaces with applications. Nonlinear Anal. 72 3929-3942 (2010)

8. Qiu, Z: Existence and uniqueness of common fixed points for two multivalued operators in ordered metric spaces. Comput. Math. Appl. 63, 1279-1286 (2012)

9. Mizoguchi, N, Takahashi, W: Fixed point theorems for multivalued mappings on complete metric spaces. J. Math. Anal. Appl. 141, 177-188 (1989)

10. Li, HG: s-coincidence and s-common fixed point theorems for two pairs of set-valued noncompatible mappings in metric space. J. Nonlinear Sci. Appl. 3, 55-62 (2010)

11. George, A, Veeramani, P: On some results in fuzzy metric spaces. Fuzzy Sets Syst. 64, 395-399 (1994)

12. George, A, Veeramani, P: On some results of analysis for fuzzy metric spaces. Fuzzy Sets Syst. 90, 365-368 (1997)

13. Hadžić, O, Pap, E: Fixed Point Theory in Probabilistic Metric Space. Kluwer Academic, Dordrecht (2001)

14. Zhu, X-H, Xiao, J-Z: Note on 'Coupled fixed point theorems for contractions in fuzzy metric spaces'. Nonlinear Anal. 74, 5475-5479 (2011)

15. Sedghi, S, Altun, I, Shobe, N: Coupled fixed point theorems for contractions in fuzzy metric spaces. Nonlinear Anal. 72, 1298-1304 (2010)

16. Choudhury, BS, Das, K, Das, P: Coupled coincidence point results for compatible mappings in partially ordered fuzzy metric spaces. Fuzzy Sets Syst. 222, 84-97 (2013)

17. Mihet, D: Fuzzy $\psi$-contractive mappings in non-Archimedean fuzzy metric spaces. Fuzzy Sets Syst. 159, 739-744 (2004)

18. Kubiaczyk, I, Sharma, S: Common coincidence point in fuzzy metric spaces. J. Fuzzy Math. 11, 1-5 (2003)

19. Hu, XQ: Common coupled fixed point theorems for contractive mappings in fuzzy metric spaces. Fixed Point Theory Appl. 2011, Article ID 363716 (2011). doi:10.1155/2011/363716

20. Cho, YJ, Sedghi, S, Shobe, N: Generalized fixed point theorems for compatible mappings with some types in fuzzy metric spaces. Chaos Solitons Fractals 39, 2233-2244 (2009)

21. Ćirić, LB: Some new results for Banach contractions and Edelstein contractive mappings on fuzzy metric spaces. Chaos Solitons Fractals 42, 146-154 (2009)

22. Mihet, D: On fuzzy contractive mappings in fuzzy metrics paces. Fuzzy Sets Syst. 158, 915-921 (2007)

23. Razani, A: A contraction theorem in fuzzy metric space. Fixed Point Theory Appl. 2005, 257-265 (2005). doi:10.1155/FPTA.2005.257

24. Gregori, V, Sapena, A: On fixed-point theorems in fuzzy metric spaces. Fuzzy Sets Syst. 125, 245-252 (2002)

25. Bhaskar, TG, Lakshmikantham, V: Fixed point theorems in partially ordered metric spaces and applications. Nonlinear Anal. 65, 1379-1393 (2006)

26. Kiany, F, Amini-Harandi, A: Fixed point and endpoint theorems for set-valued fuzzy contraction maps in fuzzy metric spaces. Fixed Point Theory Appl. 2011, Article ID 94 (2011). doi:10.1186/1687-1812-2011-94

27. Arshad, M, Shoaib, A: Fixed points of a multivalued mappings in fuzzy metric spaces. In: Proceedings of the World Congress on Engineering, vol. I. WCE 2012, July 4-6, London, U.K. (2012)

28. Gregori, V, Morillas, S, Sapena, A: Examples of fuzzy metrics and applications. Fuzzy Sets Syst. 170, 95-111 (2011)

doi:10.1186/1687-1812-2013-162

Cite this article as: Qiu and Hong: Coupled fixed points for multivalued mappings in fuzzy metric spaces. Fixed Point Theory and Applications 2013 2013:162. 